This item was submitted to Loughborough's Research Repository by the author.

Items in Figshare are protected by copyright, with all rights reserved, unless otherwise indicated.

\title{
People in pain make poorer decisions
}

\section{PLEASE CITE THE PUBLISHED VERSION}

https://doi.org/10.1097/j.pain.0000000000001542

\section{PUBLISHER}

(c) International Association for the Study of Pain. Published by Lippincott, Williams \& Wilkins

\section{VERSION}

AM (Accepted Manuscript)

\section{PUBLISHER STATEMENT}

This is a non-final version of an article published in final form in ATTRIDGE, N. ... et al., 2019. People in pain make poorer decisions. Pain, 160 (7), pp.1662-1669.

\section{LICENCE}

CC BY-NC-ND 4.0

\section{REPOSITORY RECORD}

Attridge, Nina, Jayne Pickering, Matthew Inglis, Edmund Keogh, and Christopher Eccleston. 2019. "People in Pain Make Poorer Decisions". Loughborough University. https://hdl.handle.net/2134/37095. 
Nina Attridge ${ }^{1 *}$, Jayne Pickering ${ }^{1}$, Matthew Inglis ${ }^{1}$, Edmund Keogh ${ }^{2}$ \& Christopher Eccleston ${ }^{2,3}$

${ }^{1}$ Mathematics Education Centre, Loughborough University, UK

${ }^{2}$ Centre for Pain Research, University of Bath, UK

${ }^{3}$ Department of Clinical and Health Psychology, Ghent University, Belgium

15

16

$17 *^{*}$ Corresponding author: Dr Nina Attridge, Mathematics Education Centre, Loughborough

18 University, Loughborough, Leicestershire, LE11 3TU, United Kingdom,

19

n.f.attridge@Iboro.ac.uk, +441509 223315.

20 
3 Abstract

Chronic pain affects 1 in 5 people and has been shown to disrupt attention. Here, we

5 investigated whether pain disrupts everyday decision making. In Study 1, 1322 participants completed two tasks online: a shopping decisions task and a measure of decision outcomes over the previous 10 years. Participants who were in pain during the study made more errors on the shopping task than those who were pain-free. Participants with a recurrent pain condition reported more negative outcomes from their past decisions than those without recurrent pain. In Study 2, 44 healthy participants completed the shopping decisions task with and without experimentally-induced pain. Participants made more errors while in pain than while pain-free. We suggest that the disruptive effect of pain on attending translates into poorer decisions in more complex and ecologically valid contexts, that the effect is causal, and that the consequences are not only attentional, but financial.

Keywords: Pain, cognitive disruption, decision making, finances, numeracy

\section{Summary:}

Pain had a disruptive effect on decision making in ecologically valid contexts. The effect is causal, and the consequences are not only cognitive, but financial. 


\section{Introduction}

2

3

4

5

6

7

8

9

The disruptive effect of pain on attending has been demonstrated with experimentally-induced pain[30,40], chronic pain[9,15] and transient pain such as headache[4,24,31]. This field has predominantly focused on simple cognitive processes (although sometimes using complex tasks combining multiple executive functions[25]). Few studies have examined the effects of pain on higher-level cognition. One which did, found that clinical pain was associated with less-abstract thinking[20], while another found no evidence that experimentally-induced pain affected abstract thinking[2]. Here, we focus on the potential impact of pain on higher-level real-world cognitive tasks requiring attention, namely numerical reasoning and decision making, which have serious consequences if one gets them wrong.

Reasoning and decision making are required in many areas of life and are influenced by various cognitive and emotional factors. Here, we focus on numeracy as a domain that is important in many areas of life, including budgeting, choosing a mortgage and choosing insurance plans. Despite its importance, numeracy in adults is poor. In the quantitative domain of the USA's 2003 National Assessment of Adult Literacy, 55\% of adults performed at a basic or below basic level (at best being able to locate easily-identifiable quantitative information and solve one-step arithmetic problems when the operation was specified or easily-inferred)[26]. These findings were echoed in a 2016 UK Money Advice Service study[29] into the public's ability to choose the best supermarket deals for four products. While $74 \%$ of participants chose the best deal for at least one product, only $2 \%$ chose optimally for all four.

Attention is important for learning and performing numerical operations in both children and adults[13,36]. Attention is also important in decision making, where we need 
1 to consider various options, estimate their likely outcomes, then hold these in mind while

2 choosing among them. Given that pain impairs attention, it may also influence numerical

3 decision making. Indeed, there is some initial evidence that this is the case. Placing a hand

4 into ice-cold water changed participants' risk-taking on a financial decision-making task[35].

$5 \quad$ We investigated the effect of pain on everyday decision making. In Study 1, a large

6 general population sample recruited online reported whether they were currently in pain

7 and whether they had any recurrent pain conditions. They completed two tasks: the

8 shopping decision task used by the Money Advice Service[29] and the Decision Outcomes

9 Inventory (DOI[10]), which measures real-world outcomes of everyday decisions made over

10 the previous 10 years. We hypothesized that participants who were in pain would find the

11 best shopping deal on fewer items than participants who were pain-free. If the effect of

12 pain on attention does translate into poorer decision making, the outcomes of these poor

13 decisions may accumulate in people with chronic pain. We therefore hypothesized that

14 participants with pain that had lasted for three months or longer would report more negative decision outcomes on the DOI than other participants. In Study 2 we took an experimental approach to determine a causal relationship: participants completed an extended shopping-decisions task with their hand in warm or painfully-cold water.

\section{Study 1 Method}

Design and procedure

Participants $(N=1322)$ took part online and were recruited via Amazon's Mechanical

22 Turk $(\mathrm{N}=658)$ and Prolific.ac $(\mathrm{N}=664)$. Research has shown data collected online for psychology studies is reliable[11,33] and that samples tend to be more diverse than traditional university-based samples[27]. The large sample size was decided based on 
1 previous online pain studies[3,5,7], taking into account the likelihood of substantial non-

2 completion, the noise associated with online data collection, and likely percentages of

3 participants who would report current pain (estimated at $30 \%$ ) or recurrent pain (estimated

4 at $40 \%$ ), to ensure sufficient numbers of participants in each cell (a minimum of 20 ) of the

5 current pain $x$ recurrent pain $x$ sex interaction. We achieved this aim, with the smallest cell

6 size in our primary analyses (after exclusions, see the "Data Cleaning" section) being 22

7 participants who were male with both current and recurrent pain.

Participants were directed to the study pages in Qualtrics via the recruitment

systems. After giving informed consent, participants then answered demographic questions, reported whether they had current and/or recurrent pain, completed the Decision

Outcomes Inventory and completed a shopping decisions task (see below for question and task details). A 'debrief' page appeared at the end which gave information on the purpose of the study, how to contact the researchers with any questions, and how to receive payment on MTurk or Prolific (\$2 or $£ 2$ respectively, based on the recommended per-hour rates for each recruitment site).

Participants and demographics Participants ( $\mathrm{N}=1322$ ) were 699 males, 619 females, 3 trans females and 1 trans male. Ages ranged from 18 to $74(M=33.98, S D=10.80)$. English was the first language for 1201 participants, with 16 reporting Hindi, 11 reporting Portuguese, and the remaining 94 reporting a wide variety of other languages. The majority of participants were based in the USA ( $N=799)$, followed by the UK $(N=341)$, and India $(N=44)$, with the remaining 138 reporting a variety of other countries. Participants reported their highest educational qualification, and $54 \%$ reported a university degree or above. 
2 Pain characteristics

3 Participants were asked whether they were in pain at the time of the study ("Are you

4 in pain at this very moment, from any cause?" Yes: $N=337,25 \%, \mathrm{No}: \mathrm{N}=985,75 \%)$. Those

5 who reported a current pain condition answered follow-up questions about the type,

6 intensity, and duration of their pain. Participants were then asked whether they had any

7 recurrent pain conditions that were not causing them pain at the time ("Do you have a

8 recurrent pain condition that is not causing you pain at this moment in time?" Yes $=407$,

$931 \%$, No $=915,69 \%)$. Those who reported a recurrent pain condition answered follow-up questions about the type, intensity, and duration of the pain. The type of pain was selected

11 from a list of options, separately for current and recurrent pain (see Table 1; participants were able to select multiple types of pain). Pain intensity was indicated on a numerical rating scale from 0 "No pain at all" to 10 "Worst pain imaginable" separately for current and recurrent pain (current $\mathrm{M}=3.47, \mathrm{SD}=1.79,95 \% \mathrm{Cl}[3.28,3.66]$; recurrent $\mathrm{M}=5.42, \mathrm{SD}=$ $1.83,95 \% \mathrm{Cl}[5.24,5.60]$, participants were instructed to give an average pain rating if they had more than one recurrent painful condition). Participants were asked to indicate when

17 their pain had started from a list of options (see Table 2), separately for current and recurrent pain. They were instructed to answer based on their longest standing painful condition if they had more than one type of current pain or more than one type of recurrent pain. 
$6 \quad$ Measures

7 Shopping-Decisions Task

The shopping-decision task was adapted from that used by the UK Money Advice

9 Service[29] (see https://figshare.com/s/17f3800d66375b92fb71 for the questions used

here). We used the original four questions, based on actual deals available in supermarkets,

11 and created two new ones to increase the potential variance in the data. Each question asked participants which of 4 or 5 options represented the best deal for buying a particular product. For example:

Of the following options for milk, which represents the best deal?:

a) Six pints of milk for $£ 1.80$

b) Four pints of milk for $£ 1.40$

c) Two six-pint cartons of milk on offer for $£ 3.50$

d) Two four-pint cartons of milk on offer for $£ 2$

We included a "don't know" option for each question. This was intended to serve two purposes: first, to reduce the risk of participants getting correct answers simply by guessing, and second, to reduce the risk of any participants with mathematics anxiety[21] feeling anxious about being asked to perform arithmetic, by making it clear that they could avoid it if they wished. 

total score ranging from 0 to 6 .

3

Decision Outcomes Inventory (DOI) The DOI asks participants to report whether or not they have experienced each of 41 negative decision outcomes, such as missing a flight, receiving parking tickets, or going bankrupt[10]. Thirty-five of these outcomes are preceded by filter questions asking whether participants had had the opportunity to experience the outcome. For example, the question "Have you had your electricity, cable, gas or water shut off because you didn't pay on time?" was preceded by "Have you been responsible for electricity, cable, gas or water payments?".

Scores on the DOI are weighted by the proportion of the sample who reported experiencing each outcome, with less common outcomes being weighted more heavily than more common ones on the assumption that frequency reflects severity (e.g. many more people have been lost while driving than have spent a night in jail). For example, if 500 participants have bought their own food, and 470 of these have thrown away food that went bad, the score for experiencing this negative outcome would be $1-470 / 500=.06$. Participants received a positive weighted score if they had avoided a negative outcome (e.g. +0.06 if they had bought their own food and never had to throw any away because it had gone bad), a negative weighting if they had experienced the negative outcome (e.g. - 0.06 if they had bought their own food and had to throw some away because it went bad), and no weighting if they had not had the opportunity to make the bad decision (e.g. 0 if they had never bought their own food). Therefore, lower scores indicated more and/or more severe negative outcomes from decisions. 
2 Ethics

4 Participants) Sub-Committee.

5

6 Hypotheses

We hypothesized that participants who were in pain at the time of the study would

8 find the best shopping deal on fewer items than participants who were pain free. We did

9 not expect participants with recurrent pain conditions that were not causing pain at the time to be affected on the shopping task: only current pain was hypothesized to have an

11 effect. If the effect of pain on attention does translate into poorer decision making, the outcomes of these poor decisions may accumulate in people with recurrent or chronic pain.

13 We therefore hypothesized that participants with chronic pain would report more negative

14 outcomes from their decisions on the DOI than participants without chronic pain. This was because we expected pain to have disrupted the decision-making process at the time that some of the decisions were made. We did not expect current pain to affect to DOI scores.

Study 1 Results

All analyses were two-tailed.

Data access 
Data cleaning

A total of 1559 participants accessed the study pages. Of these, 1360 gave consent and proceeded, and 1322 completed the study. Eight participants missed one or two items on the DOI, but a score was calculated excluding the missing items and their data were included.

Our analyses included participants' sex, given that females tend to report more pain than males and at a higher intensity $[18,32]$, and they also tend to have higher self-reported levels of cognitive intrusion from pain than males[3]. We therefore limited the analyses to participants reporting their sex as male or female $(N=1318$, excluding the four participants who reported other sexes).

In the shopping-decision analysis, all available data $(\mathrm{N}=1318)$ were included. In the DOI analysis, however, only participants from the USA were included. This was because the frequency and acceptability of the outcomes on the DOI may vary between countries, and scores are weighted based on the relative frequency within the sample (see Method). Therefore, including multiple countries may inappropriately skew the weightings given to different decision outcomes (e.g. if alcohol-related outcomes are substantially more common in Country A than in Country B, calculating weightings across the two countries combined would overstate the severity in Country A by making drinking appear less common that it is in that country, and understate the severity in Country B by making drinking appear more common than it is in that country). The largest subgroup of participants was from the USA ( $N=795)$, so the scoring and analysis was limited to this group. Eight negative outliers (more than 3 standard deviations below the group mean) were removed from the DOI scores, bringing the sample size to 787 . 
We checked for repeated IP addresses within the dataset, and aside from cases of participants opening the link twice but only completing the study once, we identified 15 IP addresses which appeared with two complete submissions, and one which appeared with three complete submissions. It is not possible to know whether these participants completed the study multiple times or whether they were different individuals with the same IP address (e.g. because they were in the same household, public library, university student residence, or public or university computer room). We re-analysed the data excluding these cases, and the pattern of results remained the same as reported below where they are included.

We also checked for cases where participants completed the study very quickly, which might indicate that they were not responding seriously. We chose six minutes as a cut-off time based on a drop-off in the distribution of submission durations below this point. There were 71 participants with a study duration of less than six minutes, and when these participants were excluded the pattern of results remained the same as reported below where they are included.

Effect of pain on shopping deal scores

Scores on the shopping task (number of correct answers out of 6) were entered into a 2 (current pain: yes [ $N=336]$, no [ $N=982]) \times 2$ (recurrent pain: yes [ $N=406]$, no [ $N=$ 912]) $\times 2$ (sex: female [ $N=619]$, male [ $N=699]$ ) ANCOVA with age as a covariate. Age was used as a covariate because it correlated with shopping scores, $r_{\mathrm{s}}(1318)=.097, \mathrm{p}<.001$, and differed by current (yes/no) and recurrent (yes/no) pain, both $p s<.001$. Highest qualification was correlated with shopping scores, $r_{\mathrm{s}}(1310)=.109, p<.001$, but did not differ by current (yes/no) or recurrent (yes/no) pain, $p s>.406$. Our final sample of 1318 
1 gave us $95.4 \%$ power to detect main effects and $2 \times 2$ interactions with a small effect size of

$2 \quad \eta_{p}^{2}=0.01$

3 The ANCOVA revealed a significant main effect of current pain, $F(1,1309)=5.36, p=$

$4.021, \eta_{p}^{2}=.004$, with participants who were in pain scoring lower $(M=2.86, S D=1.69,95 \%$

$5 \mathrm{Cl}[2.68,3.04])$ than those who were not in pain $(M=3.11, S D=1.85,95 \% \mathrm{Cl}[3.00,3.23]$,

6 see Figure 1). There was no significant main effect of recurrent pain, $F(1,1309)=1.19, p=$

$7.275, \eta_{p}^{2}=.001$ (recurrent pain $M=2.93, S D=1.75,95 \% \mathrm{Cl}[2.76,3.10]$; no recurrent pain $M$

$8=3.05, S D=1.99,95 \% \mathrm{Cl}[2.92,3.18])$. There was no significant main effect of sex, $F(1,1309)$

$9=2.77, p=.096, \eta_{p}^{2}=.002$, (females $M=3.08, S D=1.94,95 \% \mathrm{Cl}[2.93,3.23] ;$ males $M=$

$102.90, S D=2.04,95 \% \mathrm{Cl}[2.74,3.05])$. There were no significant interactions, all $p s>.149$.

The data show that current but not recurrent pain was associated with more errors

in finding the best shopping deal, with a small effect size. To explore the extent of this effect in terms of money spent, we calculated the average price per unit of the deals chosen by participants in the current pain and no current pain groups. These data are presented in Table 3 along with the best price available for each item. These data suggest that in general, participants would have spent more than necessary on all items, and that those in pain spent more than those not in pain on half of the items. 
Effect of pain on DOI scores For the DOI, we hypothesised that participants who had recurrent pain would score

lower than those without recurrent pain, but that current pain would not affect scores.

Therefore, participants who had current pain which had lasted for three months or longer were included in the recurrent pain group. DOI scores were analysed using a 2 (current pain: yes $[N=132]$, no $[N=655]) \times 2$ (recurrent pain: yes $[N=218]$, no $[N=569]) \times 2$ (sex: female [N = 352], male [N=435]) ANCOVA. Age and highest qualification were both related to DOI scores, $p$ s $<.001$, and age differed by recurrent pain, $p=.001$, so it was included as a covariate. Our final sample of 787 gave us $80.4 \%$ power to detect main effects and $2 \times 2$ interactions with a small effect size of $\eta_{p}{ }^{2}=0.01$. There was a main effect of recurrent pain on DOI scores, $F(1,778)=4.73, p=.030$, $\eta_{p}{ }^{2}=.006$, where those with recurrent pain scored lower $(M=13.80, S D=7.60,95 \% \mathrm{Cl}$ $[12.78,14.81])$ than those without recurrent pain $(M=15.18, S D=8.95,95 \% \mathrm{Cl}[14.45$, 15.91], see Figure 2). There was no main effect of current pain on DOI scores, $F(1,778)=$ 2.39, $p=.123, \eta_{p}^{2}=.003$ (current pain $M=13.99, S D=6.56,95 \% \mathrm{Cl}[12.87,15.12]$; no current pain $M=14.98, S D=7.27,95 \% \mathrm{Cl}[14.43,15.53])$, and no main effect of sex, $F(1,778)$ $=2.13, p=.145, \eta_{p}^{2}=.003,($ females $M=14.95, S D=8.46 ; 95 \% \mathrm{Cl}[14.07,15.84] ;$ males $M=$ 


\section{Interim discussion}

Study 1 supported our hypotheses that current pain would be associated with lower scores on the shopping task while recurrent pain would be associated with lower scores on the DOI. However, due to the cross-sectional nature of Study 1, we cannot infer a causal relationship. The effect of pain on DOI scores cannot be tested experimentally because this would require sustained pain induction over the course of 10 years. However, the effect of pain on shopping decisions can be tested experimentally, and this was done in Study 2.

\section{Study 2 Method}

Design

The experiment followed a within-participants design where participants completed shopping deal questions in two conditions: with their non-dominant hand in painful cold water $\left(8^{\circ} \mathrm{C}\right)$ and with their non-dominant hand in non-painful warm water $\left(30^{\circ} \mathrm{C}\right)$.

\section{Participants}

Participants were 44 students and members of staff at Loughborough University.

There were 32 females and 12 males, who ranged in age from 18 to $47(M=24.57, S D=$ 7.24). Our sample size was pre-determined to give us $90 \%$ power to detect an effect size of $d=0.5$ with a family-wise alpha level of 0.05 in a two-tailed paired-samples $t$-test. This effect size was chosen as the smallest effect size of interest. 
Participants completed a health screening questionnaire to ensure that they were

2 eligible for the study. Participants were ineligible for the study if they reported any of the

3 following: current pain, chronic pain, uncorrected vision problems, heart problems, a history

4 of fainting or seizures, serious cognitive impairment (e.g. dementia), asthma, a history of

5 frostbite, arthritis in their hands or forearms, a fracture in their hands or wrists, open cuts or

6 sores on their hands or wrists, a history of Reynaud's phenomenon, or having taken pain

7 killers in the previous 12 hours. These criteria were intended to ensure participants' safety

8 when taking part in the cold pressor task, and that the data would be of high quality.

9

Measures

\section{Shopping decisions task}

The shopping-decisions task was adapted for the cold pressor pain-induction paradigm used in this study. We created 90 items, which each posed a question with two answer options. For example:

Of the following options for buying grapes, which represents the best deal?

a) One 500 g pack costing $f 2.00$

b) Buy two get the third free deal on $500 \mathrm{~g}$ packs costing $£ 2.50$ each

Reducing the number of answer options meant that participants would be able to complete more questions in the timeframe allowed by the pain induction procedure, which in turn increased the sensitivity of the measure. See https://figshare.com/s/17f3800d66375b92fb71 for the full set of questions.

Six 15 -item blocks were created from the 90 items, and the blocks were presented in a fixed order. The starting condition (pain or control) was counterbalanced by participants, meaning that the items were counterbalanced by condition. The 15 items within each block 
1 were presented in a random order for each participant. If participants responded to all 15

2 items within the 90 -second time limit, the list restarted. This occurred for five participants in

3 a total of 11 blocks (69 questions, 3.2\% of the data). The analyses presented below include

4 these repeated questions, but the results remained the same when they were excluded.

5

Visual analogue scales (VASs)

After each block of the shopping task questions, participants responded to the question "How much pain were you in during the block?" by clicking on a visual analogue scale anchored by "No pain at all" on the left and "Worst pain imaginable" on the right.

\section{Procedure}

Participants took part individually in a quiet laboratory. They were sent an information sheet and health screening questionnaire prior to the study session for their reference. At the start of the session, they were asked to read the information sheet and complete a consent form, then complete the health screening questionnaire to ensure eligibility for the study (see "Participants" section for exclusion criteria).

The shopping task and VASs were presented on a desktop PC in E-Prime 3.0 with a $34 \mathrm{~cm} \times 27 \mathrm{~cm}$ monitor. The study began with a practice VAS and a practice shopping task 
1 question. Participants then completed six blocks of the shopping task, with each block

2 followed by a VAS. Each shopping task block lasted for 90 seconds, and during each one

3 participants had their non-dominant hand in either cold $\left(8^{\circ} \mathrm{C}\right.$, pain condition) or warm $\left(30^{\circ} \mathrm{C}\right.$,

4 control condition) water. The pain and control blocks alternated in order, and the starting

5 condition was counterbalanced. Due to experimenter error, one participant completed the

6 wrong counterbalanced order, meaning that 23 participants started with a pain block and

721 with a control block. In between blocks the participants had the opportunity to warm

8 their hand and only began the next block once they were pain-free. Participants were free

9 to withdraw their hand from the water at any time during a block, but none did. At the end of the study, participants were thanked, debriefed, and given $f 5$ in cash.

Study registration

In between the completion of the first study and the start of the second we introduced into our labs the scientific practice of registering study protocols prior to study initiation, in line with current recommendations[12]. Our hypothesis, sample size and analysis plan for study 2 were registered at www.aspredicted.org (\#8022; a pdf for peer review is available at http://aspredicted.org/blind.php? $x=r k 2 p 33)$. Our hypothesis was that "On a value-for-money shopping deal task, participants will give fewer correct answers AND/OR more incorrect answers while experiencing pain compared to when they are pain free." Our analysis plan was to conduct two paired-samples t-tests to see whether (a) the number of correct responses and/or (b) the number of questions attempted but incorrect differed between the pain and no pain conditions. To maintain a family-wise alpha level of 0.05, a Bonferroni correction was applied, making the alpha level 0.025 for each $t$-test. Any a posteriori analyses that were not registered are labelled as 'Exploratory' below. 
2 Ethics

3 The study received ethical approval from the Loughborough University Ethical

4 Approvals (Human Participants) sub-committee, and the University of Bath's Psychology

5 Ethics Committee and Research Ethics Approval Committee for Health.

6

$7 \quad$ Study 2 Results

8 All analyses were two-tailed.

9

Data access

Data files and analysis scripts are openly available at

\section{2 https://figshare.com/s/17f3800d66375b92fb71}

Data cleaning

In line with our study registration, we ran several checks on the data before the main analysis was conducted. These checks resulted in four participants' data being removed and

17 replaced. Two participants were removed because their average pain rating in the pain

18 blocks was not significantly different to their average pain rating in the control blocks. One participant was removed because their number of attempted but incorrect questions was more than three standard deviations from the group mean in the control condition. Finally, one participant was removed because their mean response time was more than three standard deviations from the group mean response time in the control condition. 
2 the pain and control conditions, in terms of (a) number of questions answered correctly,

3 and (b) number of questions attempted and answered incorrectly (with the alpha level

4 Bonferroni corrected to 0.025$)$. There was no effect of pain condition on number of correct

5 answers, $t(43)=0.74, p=.464$, Hedges' $g_{a v}=.095$ (Pain $M=18.89, S D=9.23,95 \% \mathrm{Cl}[16.08$,

6 21.69]; Control $M=18.14, S D=6.00,95 \% \mathrm{Cl}[16.31,19.96])$. However, there were more

7 incorrect answers in the pain condition $(M=7.43, S D=5.83,95 \% \mathrm{Cl}[5.66,9.21])$ than in the

8 control condition $(M=4.66, S D=2.47,95 \% \mathrm{Cl}[3.91,5.41]), t(43)=3.94, p<.001$, Hedges'

$9 g_{a v}=0.608$ (see Figure 3).

Exploratory analyses

VAS ratings were compared in the pain and no-pain conditions as a manipulation check. VAS ratings were higher in the pain condition $(M=63.11, S D=17.08,95 \% \mathrm{Cl}[57.92$, $68.31])$ than in the control condition $(M=3.23, S D=3.57,95 \% \mathrm{Cl}[2.15,4.32])$, and the difference was significant, $t(43)=23.27, p<.001$, Hedges' $g_{a v}=4.768$. between male and female participants, we ran a 2 (condition: pain, control) x 2 (sex: male, female) mixed ANOVA. There was no significant interaction, $F(1,42)=0.06, p=.815, \eta_{\rho}^{2}=$ .001 . 
In our main analysis we found that participants gave more incorrect answers in the pain condition than in the control condition, but no evidence that they gave fewer correct answers. This suggests that they may have answered the questions more quickly in the pain condition than in the control condition, thereby attempting more items but increasing their error rate. To test for this, we compared the total number of items attempted between the pain and control conditions. As expected, participants attempted more questions in the pain condition $(M=26.32, S D=13.60,95 \% \mathrm{Cl}[21.56,31.08])$ than in the control condition $(M=$ $22.80 S D=7.14,95 \% \mathrm{Cl}[20.30,25.29]), t(43)=2.35, p=.024$, Hedges$^{\prime} g_{a v}=0.319$

\section{Discussion}

Chronic pain patients often report difficulty in attending, and studies with both chronic and laboratory-induced pain have provided quantitative evidence that pain negatively affects performance on varieties of attention tasks. Here, we investigated for the first time whether pain disrupts performance on real-world and higher-level cognitive tasks that require attention, namely numerical and everyday decision making, which can have real and serious consequences for individuals.

In Study 1 we showed that people who were in pain at the time of completing an online shopping-decisions task made more errors than people who were not in pain at the time. Furthermore, people who reported a recurrent pain condition had experienced more negative events over the past 10 years of their lives, possibly resulting from poor decision making, than people who did not report a recurrent pain condition. In Study 2, we induced pain in healthy participants while they performed an extended version of the shopping task and found that they made more errors while they were in pain than while they were painfree, supporting our hypothesis of a causal effect of pain on shopping decisions. This effect 
1 was driven by participants attempting to answer more questions in the time allowed. We

2 found no evidence of sex differences in any of these effects.

3 Our findings extend the literature showing that pain disrupts basic attention

4 processes $[4,14,30,31,40]$ by showing that this disruptive effect translates into poorer

5 decisions in more complex and ecologically-valid contexts. This could have implications for

6 many aspects of daily life for people in pain, such as school, work, and managing finances.

7 The effects of pain on both tasks in Study 1 were small, but even a small effect of pain on

8 each of the many decisions we need to make every day can add up, over the course of

9 years, to substantially poorer quality of life in people with chronic pain. Indeed, in Study 2,

with a more sensitive measure and controlled environment, we observed a medium-sized

11 effect of pain on shopping decisions (Hedges $g_{a v}=0.608$ ).

The findings from Study 2 hint towards potential mechanisms of the effect of pain on shopping decisions. When participants were in pain, they attempted more questions and made more errors, but they did not give fewer correct answers. This is suggestive of three possible explanations. First, it could be that participants felt the need to rush through the questions when they were in pain in case it made the pain end sooner. Participants were told that every block would last for 90 seconds, but it is possible that they still consciously or subconsciously thought that going faster would make the pain end sooner. Second, it could be that pain reduces our confidence in our thinking skills so that participants were less willing to think through the arithmetic for items they found particularly difficult, and instead guessed. This would lead to more questions being attempted, and more errors being made. Third, it could be that pain increases confidence so that participants didn't check their answers whilst in pain, whereas they may have checked them if they were not in pain. This would also increase the number of items attempted in the time limit and lead to more 
1 errors. Due to participants being aware that all blocks would last for exactly 90 seconds, we suggest that the second and third explanations are more likely than the first. These are both metacognitive explanations which could be tested using established paradigms from the metacognition literature. For example, participants could be asked to indicate their initial judgment of solvability for each item, and their interim and/or final confidence in each answer they give [1]. whereby pain disrupts participants' ability to perform arithmetic calculations. If that had been the case, we would have expected fewer correct answers in the pain condition than in the control condition. Nevertheless, in many studies, pain has been associated with lower accuracies and/or slower response times on cognitive tasks. Thinking about these more general patterns of findings, we can theorise mechanisms at the cognitive level as well as at the metacognitive level. At the cognitive level, executive functions may be useful for thinking about potential mechanisms. Executive functions are the processes by which we regulate our attention, thoughts and actions [28]. When we complete a task whilst in pain, we may repeatedly switch our attention between the pain and the task, focusing on each

17 fully but for short periods, which could have engagement and disengagement costs in both

18 time and accuracy: one 'loses track'. Alternatively, the pain and the task at hand may be processed simultaneously rather than alternately. In this scenario, rather than the task suffering from disengagement costs, it suffers from a continually lower working memory capacity being available. Inhibition skills may be important in controlling our attention deployment in either of these scenarios. Additionally, there are likely motivational [39] and attentional bias [23] elements to the deployment of attention between task- and painrelated goals. 
If we can identify the mechanism(s) of the disruptive effect of pain on attention, and

2 these are found to be common across multiple tasks, then we may be able to develop

3 targeted interventions that mitigate its more extreme effects in everyday tasks that have

4 serious consequences of error [6]. Research is already making steps in this direction; Baker

5 et al [8] tested a broad programme of cognitive training as a way of improving cognition in

6 chronic pain patients, with some promising results.

In Study 1, we recruited participants reporting current pain and a history of pain.

8 Participants were blind to the task, and unaware of our hypotheses, but were not randomly

9 assigned to groups. This meant we could not test whether the relationships in question were causal, and if so, in which direction. This shortcoming was remedied for the shopping task in Study 2, where we found evidence for a causal effect of pain on shopping decisions with a true experiment. Our finding that participants with a history of pain reported more negative outcomes from real-life decisions should be conceptually replicated by giving the DOI to a clinical chronic pain sample, with participants providing a more comprehensive clinical history, and a matched control group. This would provide stronger evidence for a claim that the effect is indeed due to the participants' pain and not due to a confound. Such a study could consider how long a patient's pain has persisted, how often it occurs, and for what proportion of time (e.g. per week) they generally experience pain. We would expect that the more time a participant spends in pain per week, the greater the effect on their decision making. interpretation bias. For example, compared to people without recurrent pain, people with recurrent pain may remember past experiences in a more negative light, or have a better recollection of negative events, or be more likely to mis-remember events as having bad 
outcomes due to an expectation that their pain will have disrupted their decision making. A

2 longitudinal study regularly measuring decision outcomes in a chronic pain sample and

3 matched control group would help to differentiate these explanations from our proposal

4 that pain disrupts decision making.

$5 \quad$ These studies begin to address an important gap in our knowledge. Although

6 numeracy, reasoning and decision making are core skills embedded in almost all everyday

7 tasks, we know little about the effects of embodied distractions on them. We know that

8 time pressure [17,37], perceptual disfluency [38], and working memory load [19,22,34] can

9 detrimentally affect performance on reasoning and decision-making tasks, but the effects of

10 embodied (interoceptive or proprioceptive) distractions such as pain, itch, imbalance,

11 hunger, thirst and fatigue are less investigated [16]. In summary, we have shown that both naturally-occurring and experimentallyinduced pain is associated with poorer decisions when making grocery purchasing decisions, and that the negative effect of pain on cognition may accumulate over time in people with recurrent or chronic pain. People with pain frequently report that the cognitive consequences are fatiguing, distressing, and disruptive. Here we provide data that suggest

17 that the consequences of that disruption are not only psychological, but also financial. 
People in pain make poorer decisions 25

\section{Acknowledgments}

2 Study 1 was in part funded by an unrestricted grant from Reckitt Benckiser UK Commercial

3 Ltd to CE and EK. EK has provided unrelated consultancy services to Reckitt Benckiser UK

4 Commercial Ltd.

5

6 
2 [1] Ackerman R, Thompson VA. Meta-reasoning: monitoring and control of thinking and

3

4

5

6

7

8

9 reasoning. Trends Cogn Sci 2017:21;607-617.

[2] Agerstrom J, Gunnarsson H, Stening K. Does physical pain impair abstract thinking? J Cogn Psychol, 2017;29:748-754.

[3] Attridge N, Crombez G, Van Ryckeghem D, Keogh E, Eccleston C. The Experience of Cognitive Intrusion of Pain: scale development and validation. PAIN 2015:156;19781990.

[4] Attridge N, Eccleston C, Noonan D, Wainwright E, Keogh E. Headache impairs attentional performance: a conceptual replication and extension. J Pain 2017:18;29-41.

[5] Attridge N, Keogh E, Eccleston C. The effect of pain on task switching: pain reduces accuracy and increases reaction times across multiple switching paradigms. PAIN 2016:157;2179-93.

[6] Attridge N, Niederstrasser N. Interventions for attentional disruption in pain: cognitiongeneral, mechanism-specific, or exercise-based? PAIN 2018:159;621-622.

[7] Attridge N, Noonan D, Eccleston C, Keogh E. The disruptive effects of pain on n-back task performance in a large general population sample. PAIN 2015:156;1885-1891.

[8] Baker KS, Georgiou-Karistianis N, Lampit A, Valenzuela M, Gibson SJ, Giummarra MJJP. Computerised training improves cognitive performance in chronic pain: a participant-blinded randomised active-controlled trial with remote supervision. PAIN 2018:159;644-655.

[9] Berryman C, Stanton TR, Bowering KJ, Tabor A, McFarlane A, Moseley GL. Do people with chronic pain have impaired executive function? A meta-analytical review. Clin Psychol Rev 2014:34;563-579. 
1 [10] Bruine de Bruin W, Parker AM, Fischhoff B. Individual differences in adult decision-

2

3 [11] Buhrmester M, Kwang T, Gosling SD. Amazon's Mechanical Turk a new

4

5

6

7 source of inexpensive, yet high-quality, data? Perspect Psychol Sci 2011:6;3-5.

[12] Chambers C. The Seven Deadly Sins of Psychology. Princeton: Princeton University Press, 2017.

[13] Cragg L, Gilmore C. Skills underlying mathematics: The role of executive function in the development of mathematics proficiency. Trends Neurosci Educ 2014:3;63-68.

[14] Crombez G, Eccleston C, Baeyens F, Eelen P. Attentional disruption is enhanced by the threat of pain. Behav Res Ther 1998:36;195-204.

[15] Eccleston C. Chronic pain and distraction: an experimental investigation into the role of sustained and shifting attention in the processing of chronic persistent pain. Behav Res Ther 1995:33;391-405.

[16] Eccleston C. Embodied: The psychology of physical sensation. Oxford: Oxford University Press, 2015.

[17] Evans JSB, Curtis-Holmes J. Rapid responding increases belief bias: Evidence for the dual-process theory of reasoning. Think Reason 2005:11;382-389.

[18] Fillingim RB, King CD, Ribeiro-Dasilva MC, Rahim-Williams B, Riley JL. Sex, gender, and pain: a review of recent clinical and experimental findings. J Pain 2009:10;447-485.

[19] Gilhooly K, Logie R, Wetherick N, Wynn V. Working memory and strategies in syllogisticreasoning tasks. Mem Cognit 1993:21;115-124.

[20] Gunnarsson H, Agerström J. Clinical pain, abstraction, and self-control: being in pain makes it harder to see the forest for the trees and is associated with lower selfcontrol. J Pain Res 2018:11;1105-1114. 
1 [21] Hembree A. The Nature, Effects, and Relief of Mathematics Anxiety. J Res Math Educ

2

3 [22] Jameson TL, Hinson JM, Whitney P. Components of working memory and somatic

4

5

6 1990:12;33-46. markers in decision making. Psychon Bull Rev 2004:11;515-520.

[23] Karsdorp PA, Schrooten MG, Geenen R. Attentional control and the competition between nonpain goals and the threat of pain. Eur J Pain 2018:22;181-190.

[24] Keogh E, Cavill R, Moore DJ, Eccleston C. The effects of menstrual-related pain on attentional interference. PAIN 2014:155;821-827.

[25] Keogh E, Moore DJ, Duggan GB, Payne SJ, Eccleston C. The disruptive effects of pain on complex cognitive performance and executive control. PLoS One 2013:8(12);e83272.

[26] Kutner M, Greenberg E, Baer J. A First Look at the Literacy of America's Adults in the 21st Century, 2006. Retrieved from https://nces.ed.gov/pubsearch/pubsinfo.asp?pubid=2006470

[27] Mason W, Suri S. Conducting behavioral research on Amazon's Mechanical Turk. Behav Res Methods 2012:44;1-23.

[28] Miyake A, Friedman NP, Emerson MJ, Witzki AH, Howerter A, Wager TD. The unity and diversity of executive functions and their contributions to complex "frontal lobe" tasks: A latent variable analysis. Cogn Psychol 2000:41;49-100.

[29] Money Advice Service. Shopping offers make us spend £1,300 more per year, 2016. Retrieved from https://www.moneyadviceservice.org.uk/en/corporate/pressrelease-shopping-offers-make-us-spend-1-300-more-per-year

[30] Moore DJ, Keogh E, Eccleston C. The interruptive effect of pain on attention. Q J Exp Psychol 2012:65;565-586. 
1 [31] Moore DJ, Keogh E, Eccleston C. Headache impairs attentional performance. PAIN

2

3

4

5

6

7

8

9

10

[32] Paller CJ, Campbell CM, Edwards RR, Dobs AS. Sex-Based Differences in Pain Perception and Treatment. Pain Med 2009:10;289-299.

[33] Paolacci G, Chandler J. Inside the Turk: understanding Mechanical Turk as a participant pool. Curr Dir Psychol Sci 2014:23;184-8.

[34] Pecchinenda A, Dretsch M, Chapman P. Working memory involvement in emotionbased processes underlying choosing advantageously. Exp Psychol 2006:53;191-197.

[35] Porcelli AJ, Delgado MR. Acute stress modulates risk taking in financial decision making. Psychol Sci 2009:20;278-283.

[36] Raghubar KP, Barnes MA, Hecht SA. Working memory and mathematics: A review of developmental, individual difference, and cognitive approaches. Learn Individ Differ 2010:20;110-122.

[37] Trippas D, Handley SJ, Verde MF. The SDT model of belief bias: complexity, time, and cognitive ability mediate the effects of believability. J Exp Psychol Learn Mem Cog 2013:39;1393-1402.

[38] Trippas D, Handley SJ, Verde MF. Fluency and belief bias in deductive reasoning: new indices for old effects. Front Psychol 2014:5;1-7.

[39] Van Damme S, Legrain V, Vogt J, Crombez G. Keeping pain in mind: a motivational account of attention to pain. Neurosci Biobehav Rev 2010:34;204-213.

[40] Van Ryckeghem DM, Crombez G, Eccleston C, Liefooghe B, Van Damme S. The interruptive effect of pain in a multitask environment: an experimental investigation. J Pain 2012:13;131-138. 
2 Table 1. Type of current and recurrent pain conditions reported in the Study 1 sample.

3 Percentages are of the number of participants reporting current/recurrent pain. Participants

4 were able to select multiple options so the total is greater than $100 \%$.

5

\begin{tabular}{|c|c|c|}
\hline Type of pain & Current & Recurrent \\
\hline Arthritis & $45(12 \%)$ & $61(15 \%)$ \\
\hline Back (non-muscular) & $90(27 \%)$ & $103(26 \%)$ \\
\hline Lower back & $59(18 \%)$ & $63(16 \%)$ \\
\hline Upper back & 57 (17\%) & $42(10 \%)$ \\
\hline Hangover & $7(2 \%)$ & $19(5 \%)$ \\
\hline Headache & $90(27 \%)$ & $89(22 \%)$ \\
\hline Joint & $69(21 \%)$ & $86(21 \%)$ \\
\hline Menstrual & $24(7 \%)$ & $62(15 \%)$ \\
\hline Muscular (non-back) & $27(8 \%)$ & $21(5 \%)$ \\
\hline Migraine & $12(4 \%)$ & $60(15 \%)$ \\
\hline Nerve & $24(7 \%)$ & $21(5 \%)$ \\
\hline Post-surgical & $5(1 \%)$ & $9(2 \%)$ \\
\hline Sciatica & $21(6 \%)$ & $33(8 \%)$ \\
\hline Stomach & $33(10 \%)$ & $44(11 \%)$ \\
\hline Throat & $11(3 \%)$ & $6(1 \%)$ \\
\hline Tooth & $39(12 \%)$ & 39 (10\%) \\
\hline Other & $37(11 \%)$ & $33(8 \%)$ \\
\hline
\end{tabular}


1 Table 2. Duration of current and recurrent pain conditions reported in the Study 1 sample.

2

\begin{tabular}{lcc}
\hline Duration of pain & Current & Recurrent \\
\hline Up to one hour & $39(12 \%)$ & - \\
Up to 24 hours & $71(21 \%)$ & - \\
Up to a week & $47(14 \%)$ & $18(4 \%)$ \\
Up to a month & $28(8 \%)$ & $21(5 \%)$ \\
Up to 3 months & $18(5 \%)$ & $22(5 \%)$ \\
Up to 6 months & $12(4 \%)$ & $23(6 \%)$ \\
Up to a year & $45(13 \%)$ & $78(19 \%)$ \\
Up to a decade & $52(15 \%)$ & $146(36 \%)$ \\
Over a decade & $24(7 \%)$ & $93(23 \%)$
\end{tabular}

3

4

5 
1 Table 3. Mean amount of money spent per unit for each item in the shopping decision task

2 in the current pain and no current pain groups in Study 1.

3

\begin{tabular}{lllll}
\hline & Best price & No current & Current pain & Pain/no pain \\
& available & pain average & average spend & spend \\
& & spend & & difference \\
\hline Milk (per pint) & $25 p$ & $27 p$ & $27 p$ & $0 p$ \\
Lemons (per kilo) & $f 2.10$ & $£ 2.26$ & $£ 2.27$ & $1 p$ \\
Ketchup (per kilo) & $f 2.45$ & $£ 2.61$ & $£ 2.65$ & $4 p$ \\
Eggs (each) & $11 p$ & $12 p$ & $12 p$ & $0 p$ \\
Cheese (per kilogram) & $f 9.20$ & $£ 9.64$ & $£ 9.67$ & $3 p$ \\
Cola (per litre) & $75 p$ & $80 p$ & $80 p$ & $0 p$
\end{tabular}

4 
3 Figure 1. Shopping decision scores in Study 1 by current (yes/no) and recurrent (yes/no)

4 pain with age as a covariate. There was a significant main effect of current pain, but no

5 effect of recurrent pain and no interaction. Error bars show \pm 1 standard error of the mean.

6

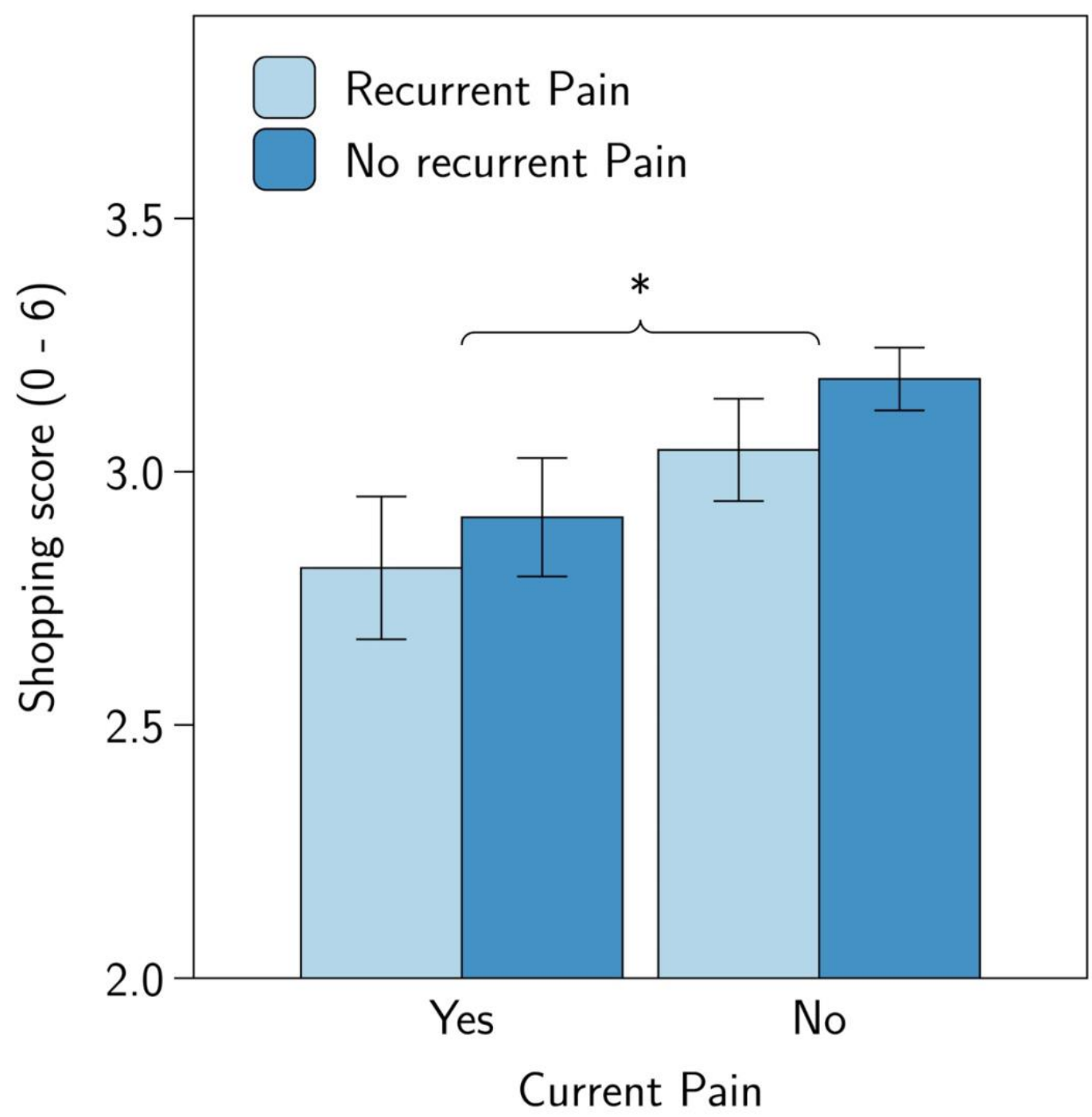


1 Figure 2. Decision Outcome Inventory scores in Study 1 by current (yes/no) and recurrent

2 (yes/no) pain with age as a covariate. There was a significant main effect of recurrent pain,

3 but no effect of current pain and no interaction. Error bars show \pm 1 standard error of the 4 mean.

5

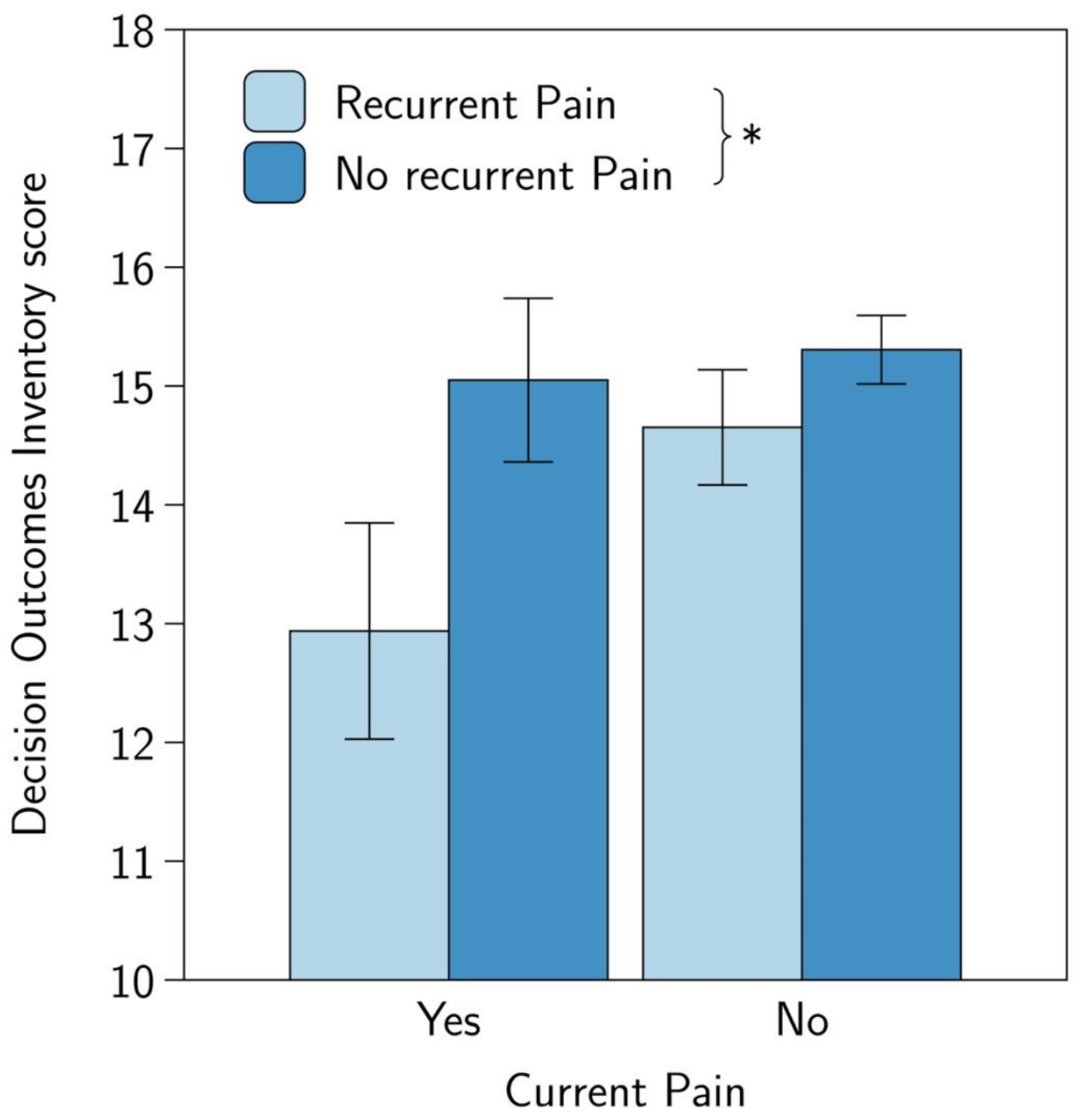

6 
1 Figure 3. Violin plot showing distributions and means of number of errors and number of

2 correct answers on the shopping decisions task by pain condition in Study 2. There was a

3 significant main effect of pain condition on errors but not on number of correct answers.

4 Error bars show \pm 1 standard error of the mean.

5

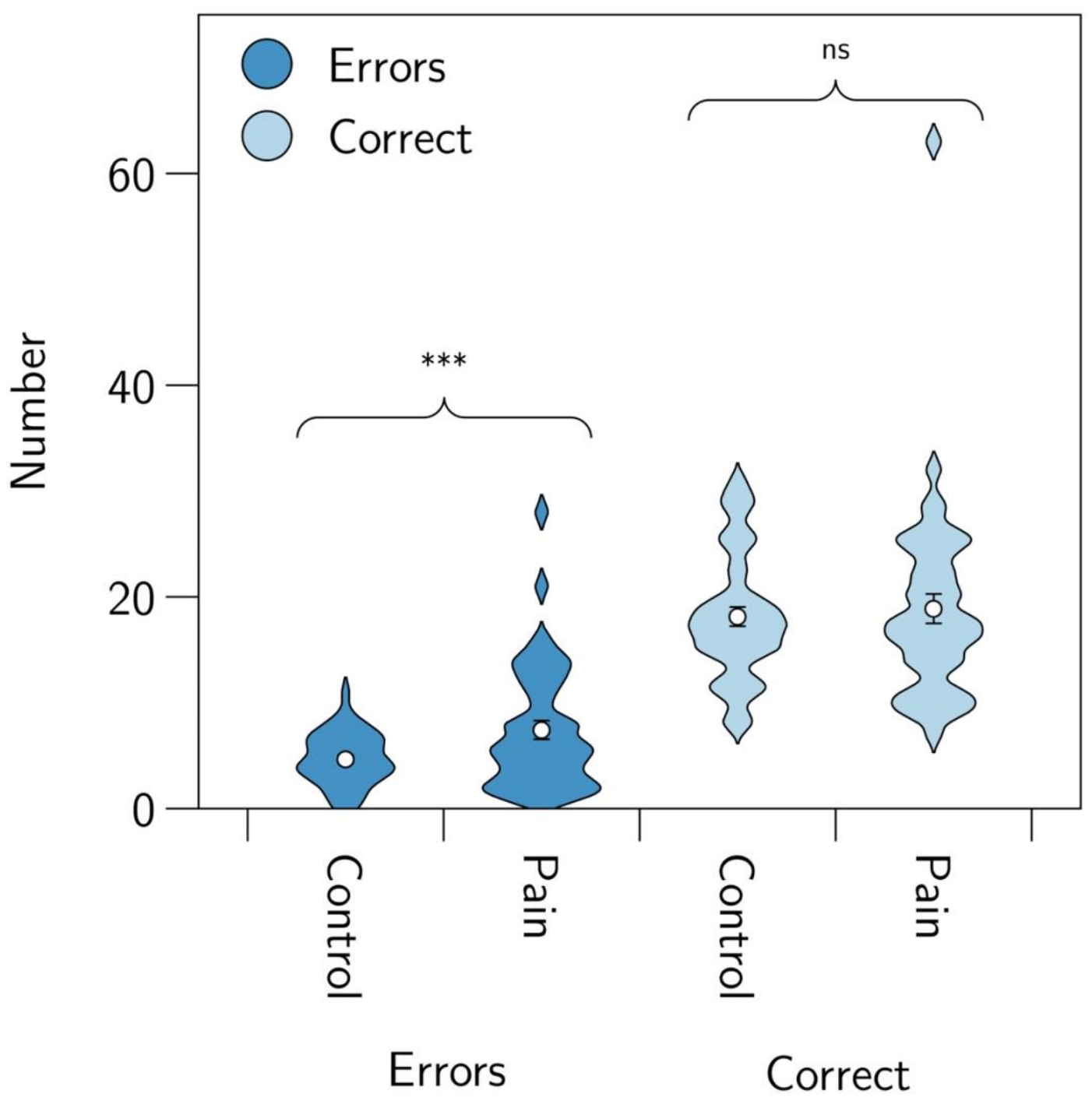

